\title{
Renalase as a Novel Biomarker for Evaluating the Severity of Hepatic Ischemia-Reperfusion Injury
}

\author{
Huili Li, ${ }^{1}$ Jianrong Guo, ${ }^{1}$ Hongli Liu, ${ }^{2}$ Yanfeng Niu, ${ }^{1}$ Lixia Wang, \\ Kun Huang, ${ }^{4}$ and Jiliang Wang ${ }^{1}$ \\ ${ }^{1}$ Department of Gastrointestinal Surgery, Union Hospital, Tongji Medical College, Huazhong University of \\ Science and Technology, 1277 Jiefang Avenue, Wuhan, Hubei, China \\ ${ }^{2}$ Cancer Center, Union Hospital, Tongji Medical College, Huazhong University of Science and Technology, 1277 Jiefang Avenue, \\ Wuhan, Hubei, China \\ ${ }^{3}$ Department of Radiology, Union Hospital, Tongji Medical College, Huazhong University of Science and Technology, \\ 1277 Jiefang Avenue, Wuhan, Hubei, China \\ ${ }^{4}$ Institution of Cardiology, Union Hospital, Tongji Medical College, Huazhong University of Science and Technology, \\ 1277 Jiefang Avenue, Wuhan, Hubei, China
}

Correspondence should be addressed to Kun Huang; kun_huang@hust.edu.cn and Jiliang Wang; jiliang_wang@hust.edu.cn

Received 13 June 2016; Revised 16 September 2016; Accepted 27 September 2016

Academic Editor: Pablo Muriel

Copyright (C) 2016 Huili Li et al. This is an open access article distributed under the Creative Commons Attribution License, which permits unrestricted use, distribution, and reproduction in any medium, provided the original work is properly cited.

\begin{abstract}
Hepatic ischemia-reperfusion (I/R) injury is a serious complication in clinical practice. However, no efficient biomarkers are available for the evaluation of the severity of I/R injury. Recently, renalase has been reported to be implicated in the I/R injury of various organs. This protein is secreted into the blood in response to increased oxidative stress. To investigate the responsiveness of renalase to oxidative stress, we examined the changes of renalase in cell and mouse models. We observed a significant increase of renalase expression in HepG2 cells in a time- and dose-dependent manner when treated with $\mathrm{H}_{2} \mathrm{O}_{2}$. Renalase expression also increased significantly in liver tissues that underwent the hepatic I/R process. The increased renalase levels could be efficiently suppressed by antioxidants in vitro and in vivo. Furthermore, serum renalase levels were significantly increased in the mouse models and also efficiently suppressed by antioxidants treatment. The variation trends are consistent between renalase and liver enzymes in the mouse models. In conclusion, renalase is highly sensitive and responsive to oxidative stress in vitro and in vivo. Moreover, renalase can be detected in the blood. These properties make renalase a highly promising biomarker for the evaluation of the severity of hepatic I/R injury.
\end{abstract}

\section{Introduction}

Ischemia is ceasing of blood supply, causing a shortage of oxygen; reperfusion is restoring of blood supply after ischemia. The ischemia-reperfusion $(\mathrm{I} / \mathrm{R})$ process occurs in many clinically important events, including hepatic resectional surgery, transplantation, trauma, and hemorrhagic shock [1-3]. Hepatic I/R injury is an inevitable complication causing severe cellular death, tissues damage, and liver dysfunction, which increases the mortality [4-6]. Reactive oxygen species (ROS), which are the main toxicants in oxidative stress, play critical roles in hepatic I/R injury [7-10].
Vascular endothelial cells, hepatic sinusoidal endothelial cells, Kupffer cells, or polymorphonuclear leukocytes are activated by the I/R process and produce a large amount of ROS in oxidative stress process, including superoxide, hydrogen peroxide $\left(\mathrm{H}_{2} \mathrm{O}_{2}\right)$, hydroxyl radicals, and nitric oxide. This ROS production is the main factor responsible for damaging liver parenchymal cells, increasing vascular permeability, and inducing inflammatory cell infiltration $[2,11-15] . \mathrm{H}_{2} \mathrm{O}_{2}$ is the most abundant and stable ROS. It leads to oxidative stress and is implicated in a variety of inflammatory diseases $[16,17]$. $\mathrm{H}_{2} \mathrm{O}_{2}$ can be converted into hydroxyl radicals, which are extremely reactive and more toxic than other $\operatorname{ROS}[8,9]$. 
In this context, $\mathrm{H}_{2} \mathrm{O}_{2}$ is the perfect agent to establish an oxidative stress model in cells and also to simulate I/R injury in vitro to some extent.

It has been proposed that I/R injury is an intricate process in which the oxidant/antioxidant balance is changed in favor of the oxidants [18]. Treatment with superoxide dismutase (SOD) and catalase (CAT) reduces the ROS levels, as SOD rapidly dismutases the superoxide $\left(\mathrm{O}_{2}{ }^{-}\right)$to $\mathrm{H}_{2} \mathrm{O}_{2}$, and CAT scavenges $\mathrm{H}_{2} \mathrm{O}_{2}$ to produce water, which reduces the severity of oxidative stress [5]. Beside SOD and CAT, a variety of compounds and biomaterials has been developed as potential therapeutic agents for I/R injury $[6,17]$. However, it still lacks efficient, specific, and sensitive biomarkers for the accurate evaluation of the severity of oxidative stress in hepatic I/R injury [19].

Renalase, a ubiquitous flavin adenine dinucleotide-containing amino oxidase, has been implicated in the process of I/R injury [20]. Since first being identified in 2005, renalase has been reported to be synthesized in various organs, including kidney, heart, liver, and adipose tissues [21]. Renalase is secreted into the blood in response to increased oxidative stress [22-24]. The elevated renalase level under stress conditions makes renalase a potential biomarker for the evaluation of the severity of organ I/R injury.

In the present study, we demonstrated that renalase is a sensitive ROS-responsive gene in hepatocytes. In hepatic $\mathrm{I} / \mathrm{R}$ injury mouse models, renalase was augmented in liver and blood. Moreover, the augmentation of renalase can be ameliorated by antioxidants pretreating, which can reduce the severity of oxidative stress, in vitro and in vivo. These findings provide evidence that renalase can serve as an efficient and sensitive biomarker for the early warning or evaluation of the severity of hepatic I/R injury.

\section{Material and Methods}

2.1. Ethics Statement. All animal experiments were performed in accordance with the National Institutes of Health (NIH) Guide for the Care and Use of Laboratory Animals published by the US National Institutes of Health $(\mathrm{NIH}$ Publication, 8th edition, 2011) and approved by the Ethics Committee of Tongji Medical College, Huazhong University of Science and Technology, China.

2.2. Reagents and Cell Culture. $\mathrm{H}_{2} \mathrm{O}_{2}$, pentobarbital sodium, SOD, and CAT were purchased from Sigma-Aldrich (St. Louis, MO, USA). Human hepatocellular carcinoma cell line HepG2 was purchased from the American Type Culture Collection (ATCC, Manassas, VA, USA). Cells were maintained in DMEM (high glucose; Invitrogen, Madison, WI, USA) supplemented with 10\% FBS (HyClone, Logan, UT, USA) and $100 \mathrm{U} / \mathrm{mL}$ penicillin- $100 \mu \mathrm{g} / \mathrm{mL}$ streptomycin (Gibco, Carlsbad, CA, USA) and kept in a humidified atmosphere at $37^{\circ} \mathrm{C}$ with $5 \% \mathrm{CO}_{2}$ in an incubator (Thermo Fisher Scientific Inc., Waltham, MA, USA).

2.3. In Vivo Hepatic I/R Model. The in vivo hepatic I/R model was performed as previously described [3, 16, 17, 25]. Male C57BL/6 mice, aged 8-12 weeks, were purchased from Beijing
University (Beijing, China) and maintained on a chow diet in a $12 \mathrm{~h}$ light $/ 12 \mathrm{~h}$ dark environment at $25^{\circ} \mathrm{C}$ in the Animal Care Facility of Tongji Medical College. Surgical procedures on mice were performed under sterile conditions by administration of pentobarbital sodium $(50 \mathrm{mg} / \mathrm{kg})$ by an intraperitoneal injection. One hour before the pentobarbital sodium anesthesia, the I/R+SOD+CAT group was intraperitoneally injected with $300 \mathrm{KU} / \mathrm{kg}$ SOD and $60 \mathrm{mg} / \mathrm{kg}$ CAT, whereas the sham and I/R mice groups were given physiological saline as the solvent by the same method. Laparotomy was performed by vertically opening $2.5-3 \mathrm{~cm}$ in the anterior part of the abdomen of the anesthetized mice. After identifying the portal triad and biliary tree, the main trunk of the hepatic artery and portal vein, except for the vasculatures to the right lower lobe, was clamped with a vascular clip to achieve ischemic injury to approximately $70 \%$ of the liver. After $1 \mathrm{~h}$ of ischemia, reperfusion was achieved by releasing the vascular clip. No vascular clamp was done for the sham group of mice. Then, the incision was closed with silk suture. Six hours after reperfusion, hepatic lobes underwent I/R and the corresponding hepatic lobes from the mice of the sham group were removed and used for further assays. Histological evaluations (H\&E staining and IHC of cleaved caspase3) were performed to quantify the degree of liver injury. Confocal immunofluorescence imaging of frozen sections was performed to detect the renalase levels. Western blotting and real-time qPCR were performed to detect the protein levels and mRNA expression of renalase in liver tissue. Blood was taken by eyeball extirpating and then centrifuged for serum separation, and the serum was used for detection of levels of the renalase and liver enzymes.

2.4. Western Blot Analysis. As previously described [26-28], total cells and tissues were lysed using RIPA lysis buffer, and the protein concentration was determined with a BCA protein assay kit (Pierce Company, Rockford, IL, USA). Protein extracts were used for SDS-PAGE (Invitrogen, Carlsbad, CA, USA), and the proteins were transferred to a polyvinylidene fluoride membrane (Millipore, Billerica, MA, USA), which was blocked with 5\% nonfat milk in TBS for $3 \mathrm{~h}$ and incubated with various primary antibodies overnight at $4^{\circ} \mathrm{C}$. After incubation with HRP-conjugated secondary antibodies (diluted 1:5000) for $1 \mathrm{~h}$ at room temperature, the membranes were treated with ECL reagents (170-5061, Bio-Rad, Hercules, CA, USA) prior to visualization using a ChemiDoc MP imaging analysis system (Bio-Rad, Hercules, CA, USA) according to the manufacturer's instructions. The specific protein expression levels were normalized to $\beta$ tubulin on the same nitrocellulose membrane. The following primary antibodies and dilutions were used: anti-renalase (GTX89570, diluted 1:1000) was purchased from GeneTex (Irvine, CA, USA); anti- $\beta$-tubulin (sc-9104, diluted $1: 2000$ ) was purchased from Santa Cruz Biotechnology (Santa Cruz, CA, USA).

2.5. Real-Time RT-RCR. As previously described [29, 30], total RNA was isolated using TRIzol reagent (Invitrogen, Carlsbad, CA, USA) according to the manufacturer's instructions. $2 \mu \mathrm{g}$ of total RNA was reversely transcripted using an 
TABLE 1: The sequences of primers for real-time qPCR.

\begin{tabular}{lccl}
\hline Name & Use & Orientation & Sequence \\
\hline \multirow{2}{*}{ Renalase } & Real-time qPCR & $\mathrm{F}$ & $5^{\prime}$-AGTGAACGCCAGAGGGAGCAA-3' \\
& & $\mathrm{R}$ & $5^{\prime}$-TAGCGGCAGGACCAAGGGAC-3 \\
$\beta$-Tubulin & $\mathrm{F}$ & $5^{\prime}$-GCTCATCGCTTATCACCTCC-3' \\
& Real-time qPCR & $\mathrm{R}$ & $5^{\prime}$-GAGCGCTCTGTCCACGTACT-3 \\
\hline
\end{tabular}

RNA PCR Kit (Takara Biotechnology, Otsu, Japan), and the resulting cDNA was used as a PCR template. The mRNA levels were determined by real-time qPCR with an ABI PRISM 7900 Sequence Detection System (Applied Biosystems, Foster City, CA, USA) according to the manufacturer's instructions, and $\beta$-tubulin was used as endogenous control. The experiment was performed in triplicate. The relative gene expression levels were calculated using the comparative $\mathrm{C}_{\mathrm{T}}$ method applying the formula $2^{-\Delta \Delta \mathrm{C}_{\mathrm{T}}}$. The primer sequences for real-time qPCR are listed in Table 1.

2.6. Confocal Immunofluorescence. Fresh ischemia-reperfusion hepatic lobes were collected and rinsed in saline to remove remaining blood. Tissues were cut into $6-\mu \mathrm{m}$ thick sections with a freezing slicing microtome. The sections were then immersed and fixed in $4 \%$ paraformaldehyde at room temperature for one-half hour. Afterwards, the sections were incubated with $5 \%$ bovine serum albumin (BSA) and immunolabeled with the indicated antibody (renalase GTX89570, GeneTex) at $4^{\circ} \mathrm{C}$ overnight. After washing, the sections were incubated with Alexa Fluor 594 goat antirabbit IgG (R37117, Invitrogen) for $1 \mathrm{~h}$. After washing, 4',6diamidino-2-phenylindole (DAPI) was added to stain the cell nuclei on ice. Tissue fluorescence was imaged on a confocal microscope (Alsi, Nikon). For the quantitative expression of renalase, the density of fluorescence was analyzed by the ImageJ $1.44 \mathrm{p}$ software.

2.7. Liver Histology and IHC. Formalin-fixed liver specimens were embedded in paraffin blocks and cut into $5 \mu \mathrm{m}$ sections. The sections were then stained with hematoxylin and eosin for histology. For IHC, the sections were deparaffinized and rehydrated with ethanol and xylene and were heated to $95-98^{\circ} \mathrm{C}$ for $20 \mathrm{~min}$ in $10 \mathrm{mM}$ citrate buffer, $\mathrm{pH}$ 6.0. After blocking with PBS containing $10 \%$ goat serum for $1 \mathrm{~h}$ at room temperature, the sections were incubated with a primary antibody 1:200 cleaved caspase- 3 overnight at $4^{\circ} \mathrm{C}$. After incubation with secondary antibodies, the sections were mounted and evaluated with an Olympus microscope.

2.8. Detection of Liver Enzymes in Serum. Alanine transaminase (ALT), aspartate transaminase (AST), gamma-glutamyl transpeptidase (GGT), alkaline phosphatase (ALP), and lactate dehydrogenase $(\mathrm{LDH})$ in serum were determined using automatic biochemical analyzer (TBA-40FR, Toshiba, Tokyo, Japan). Related detection kits were purchased from MedicalSystem Biotechnology Co., Ningbo, China.
2.9. Detection of Serum Renalase Protein Levels. The protein levels of renalase in the serum were determined by using a Mouse Renalase (RNLS) ELISA Kit (MU30925, Bio-Swamp, Wuhan, China) according to the manufacturer's instructions. Briefly, dilute standard first. Add serum $40 \mu \mathrm{L}$ to testing well, then add biotinylated anti-RNLS-antibody $10 \mu \mathrm{L}$, and gently mix. Incubate for $30 \mathrm{~min}$ at $37^{\circ} \mathrm{C}$. Dry and wash every well. Add HRP-Conjugate Reagent $50 \mu \mathrm{L}$ to each well, except the blank well. Incubate and wash. Then add chromogen solutions and incubate for $15 \mathrm{~min}$ at $37^{\circ} \mathrm{C}$. Measure the optical density (OD) at $450 \mathrm{~nm}$ after adding Stop Solution within $15 \mathrm{~min}$. Calculate according to the manufacturer's instructions.

2.10. Statistical Analysis. As described in previous studies $[31,32]$, the statistical analysis was performed with the Statistical Package for Social Sciences (SPSS version 13.0; IBM Analytics, Chicago, IL, USA). All the data were expressed as mean \pm SD (standard deviation, SD) and the difference was analyzed by a one-way ANOVA test. Statistical analysis was performed using Student's $t$-test for paired data. The difference was considered statistically significant for $p<0.05$.

\section{Results}

3.1. Increase of the Renalase Expression in HepG2 Cells Induced by $\mathrm{H}_{2} \mathrm{O}_{2}$. To investigate the impact of oxidative stress on the renalase expression in HepG2 cells, the cells were incubated with $\mathrm{H}_{2} \mathrm{O}_{2}$ in different concentrations and for different times. First, HepG2 cells were incubated with $\mathrm{H}_{2} \mathrm{O}_{2}$ in increasing concentrations $(0 \mu \mathrm{M}, 100 \mu \mathrm{M}, 200 \mu \mathrm{M}, 500 \mu \mathrm{M}$, and $1000 \mu \mathrm{M}$ ) for $6 \mathrm{~h}$, respectively. With increasing $\mathrm{H}_{2} \mathrm{O}_{2}$ concentration, the mRNA expression of renalase increased proportionally (Figure 1(a)). The maximal expression of renalase was detected for a concentration of $500 \mu \mathrm{M}$. Renalase expression decreased slightly when incubated with a concentration of $1000 \mu \mathrm{M}$ compared with $500 \mu \mathrm{M}$ (Figure 1(a)). This suggests that the concentration of $1000 \mu \mathrm{M}$ may be beyond the cell's affordable range and causes cell death. We used relatively higher concentrations $(1000 \mu \mathrm{M}, 2000 \mu \mathrm{M}, 3000 \mu \mathrm{M}$, and $5000 \mu \mathrm{M})$ of $\mathrm{H}_{2} \mathrm{O}_{2}$ to treat cells and found that cell death increased with the increasing concentrations of $\mathrm{H}_{2} \mathrm{O}_{2}$. Treating with $2000 \mu \mathrm{M}$ of $\mathrm{H}_{2} \mathrm{O}_{2}$ for $6 \mathrm{~h}$ could even cause more than half of the cell death. No cells could survive in the concentration of $5000 \mu \mathrm{M} \mathrm{H}_{2} \mathrm{O}_{2}$ (data not shown). Then, HepG2 cells were incubated with $500 \mu \mathrm{M} \mathrm{H}_{2} \mathrm{O}_{2}$ for different times ( $15 \mathrm{~min}, 30 \mathrm{~min}, 1 \mathrm{~h}, 3 \mathrm{~h}, 6 \mathrm{~h}, 12 \mathrm{~h}$, and $24 \mathrm{~h}$ ). Increasing the incubation time induced a proportional increase of the 


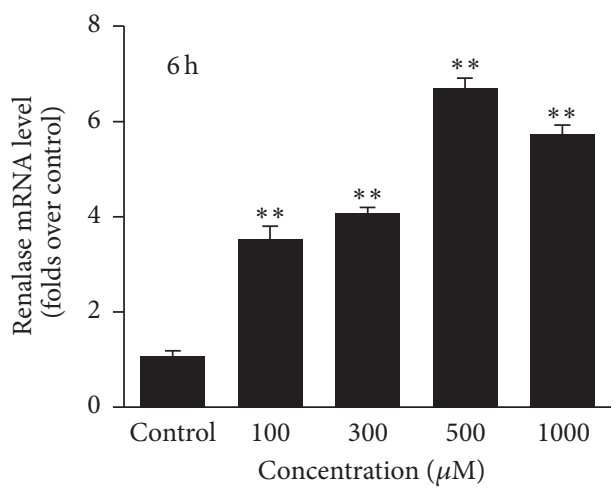

(a)

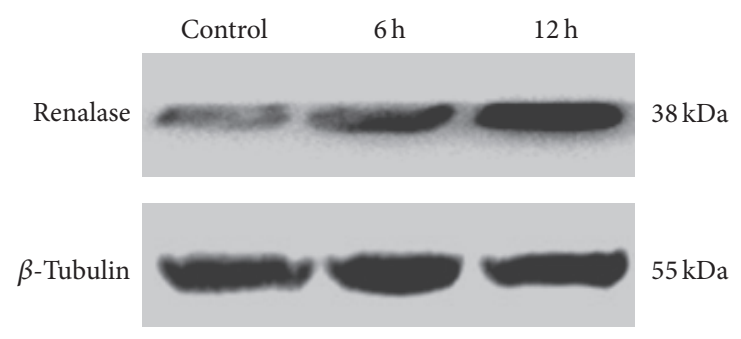

(c)

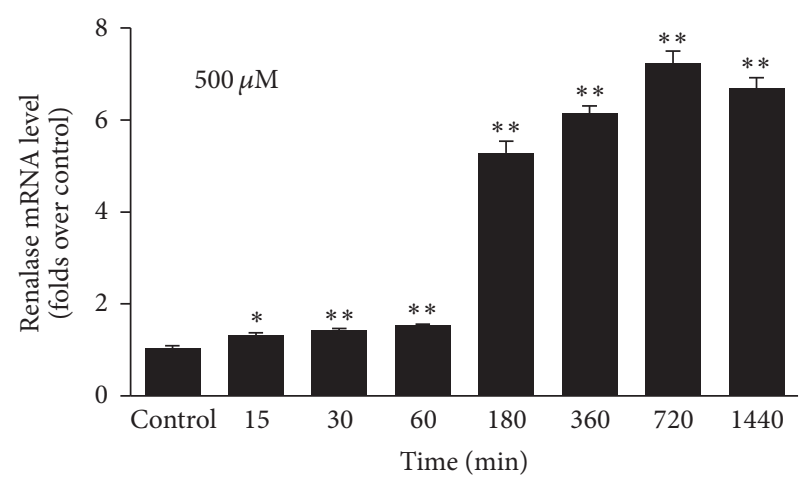

(b)

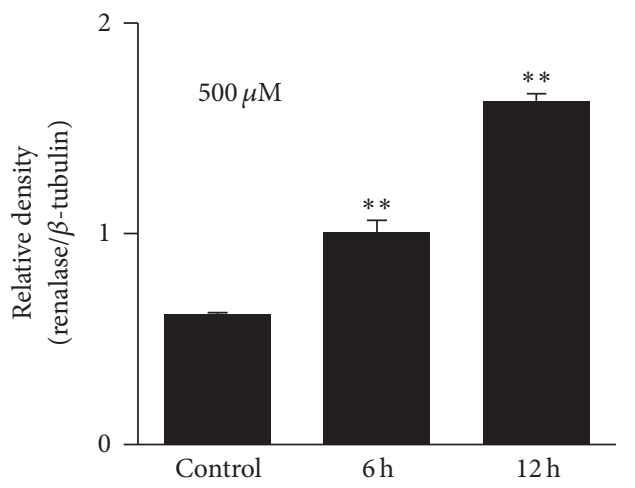

(d)

FIGURE 1: Increase of renalase expression in hepatic cells upon $\mathrm{H}_{2} \mathrm{O}_{2}$ treatment. (a) Relative expression of renalase mRNA evaluated by realtime qPCR in HepG2 cells treated with increasing concentrations of $\mathrm{H}_{2} \mathrm{O}_{2}$. (b) Relative expression of renalase mRNA evaluated by real-time qPCR in HepG2 cells treated with $\mathrm{H}_{2} \mathrm{O}_{2}$ for different times. (c) Western blot analysis of renalase protein levels in HepG2 cells treated with $500 \mu \mathrm{M} \mathrm{H}_{2} \mathrm{O}_{2}$ for $6 \mathrm{~h}$ or $12 \mathrm{~h}$. (d) Densitometric analysis of Western blot of renalase in HepG2 cells treated with $500 \mu \mathrm{M} \mathrm{H}_{2} \mathrm{O}_{2}$ for $6 \mathrm{~h}$ or $12 \mathrm{~h}$. ${ }^{*} p<0.05{ }^{* *} p<0.01$, compared with control group. Data are plotted as the mean \pm SD from five independent experiments. Bars indicate the standard deviation of the mean.

mRNA expression of renalase (Figure 1(b)). The maximal expression of renalase was measured for an incubation time of $12 \mathrm{~h}$. Renalase expression decreased slightly when incubated for $24 \mathrm{~h}$ (Figure 1(b)). This indicates that the cells may not tolerate the long-time incubation of $\mathrm{H}_{2} \mathrm{O}_{2}$. Further, the protein levels of renalase in HepG2 cells that were incubated with $500 \mu \mathrm{M} \mathrm{H}_{2} \mathrm{O}_{2}$ for either $6 \mathrm{~h}$ or $12 \mathrm{~h}$ were measured and found to be larger for the longer incubation $(p<0.01$, Figures $1(\mathrm{c})$ and $1(\mathrm{~d}))$.

3.2. Suppression of $\mathrm{H}_{2} \mathrm{O}_{2}$-Induced Augmentation of the Renalase Expression in HepG2 Cells Mediated by Antioxidant Preincubation. Antioxidants can reduce the severity of oxidative stress. To investigate whether the $\mathrm{H}_{2} \mathrm{O}_{2}$-induced augmentation of the renalase expression in HepG2 cells can be decreased by antioxidants, cells were preincubated with $300 \mathrm{KU} / \mathrm{L}$ SOD and $60 \mathrm{mg} / \mathrm{L}$ CAT for $2 \mathrm{~h}$. Before the $\mathrm{H}_{2} \mathrm{O}_{2}$-treatment, the SOD and CAT-containing medium was replaced by FBS-free medium. The obtained results showed that the SOD/CAT preincubation significantly decreased the $\mathrm{H}_{2} \mathrm{O}_{2}$-induced augmentation of the renalase mRNA expression in HepG2 cells $(p<0.01$, Figure $2(\mathrm{a}))$. Furthermore, the
SOD/CAT preincubation significantly decreased the $\mathrm{H}_{2} \mathrm{O}_{2}$ induced increase of the renalase protein levels in HepG2 cells $(p<0.01$, Figures 2(b) and 2(c)).

3.3. Increased Renalase Expression and Histologic Damage in Hepatic I/R Injury Mouse Models and the Suppression to the Increased Renalase Expression by Antioxidants. In hepatic I/R injury mouse models, the renalase mRNA expression in livers was significantly augmented $(p<0.01$, Figure $3(a))$, and the intraperitoneal preinjection of SOD and CAT decreased this augmentation significantly ( $p<0.01$, Figure 3(a)). The renalase protein levels in livers of the mouse models were significantly increased $(p<0.01$, Figure 3(c)) and could also be suppressed by the intraperitoneal preinjection of SOD and CAT $(p<0.01$, Figure 3(c)). This result was confirmed by Western blot (Figures 3(b) and 3(c)) and confocal immunofluorescence imaging (Figure 4(b)). The histological evaluation revealed that $\mathrm{I} / \mathrm{R}$ caused hydropic degeneration and necrosis of hepatic tissue and cells. A significant improvement was observed in $\mathrm{I} / \mathrm{R}+\mathrm{SOD}+\mathrm{CAT}$ group (Figure $4(\mathrm{a})$ ). Consistent with the observed hydropic degeneration and necrosis during $\mathrm{I} / \mathrm{R}$, hepatic apoptosis was increased as 


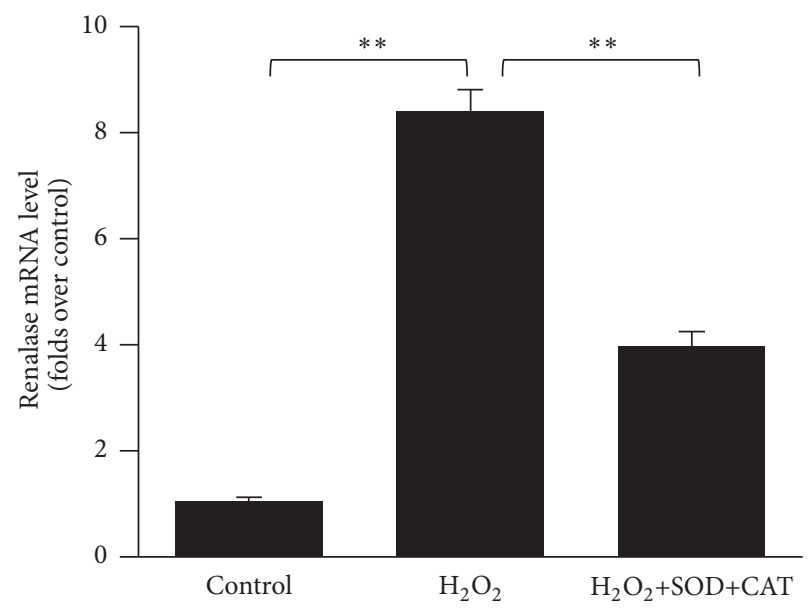

(a)

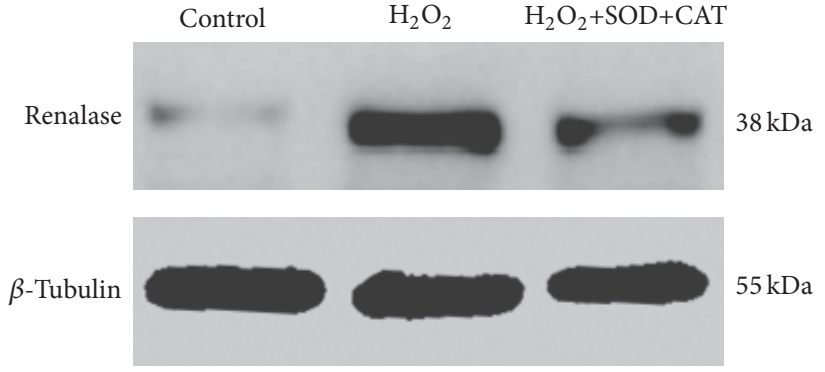

(b)

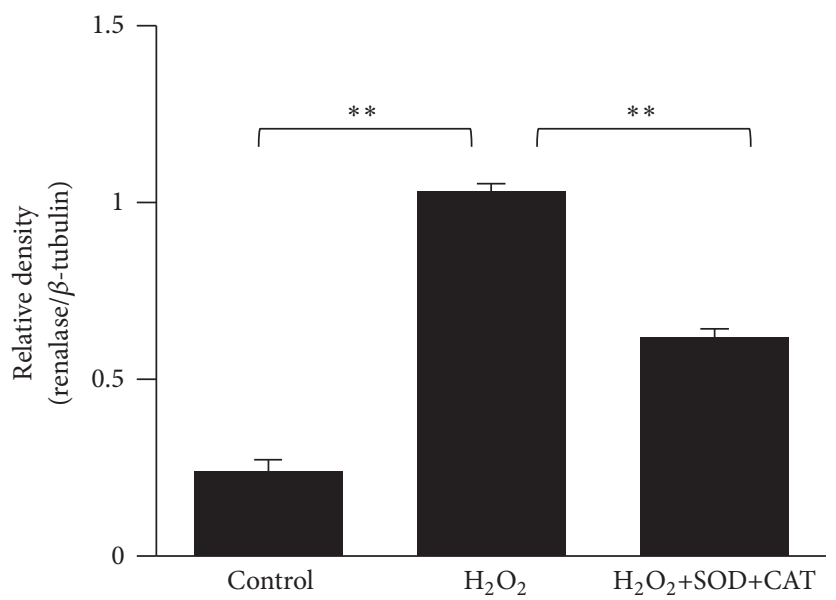

(c)

FIGURE 2: Antioxidant-induced decrease of $\mathrm{H}_{2} \mathrm{O}_{2}$-induced augmentation of the renalase expression in hepatic cells. (a) Relative expression of renalase mRNA evaluated by real-time qPCR in $\mathrm{H}_{2} \mathrm{O}_{2}$-incubated ( $12 \mathrm{~h}$ ) HepG2 cells with or without pretreatment with antioxidants. (b) Western blot analysis of renalase protein levels in $\mathrm{H}_{2} \mathrm{O}_{2}$-incubated (12 h) HepG2 cells with or without preincubation with antioxidants. (c) Densitometric analysis of Western blot of renalase in $\mathrm{H}_{2} \mathrm{O}_{2}$-incubated HepG2 cells with or without preincubation with antioxidants. ${ }^{* *} p<$ 0.01 . Data are plotted as the mean \pm SD from five independent experiments. Bars indicate the standard deviation of the mean.

seen by the brown intracellular staining of cleaved caspase3 in most hepatocytes. A significant reduction of cleaved caspase- 3 staining was observed in the $\mathrm{I} / \mathrm{R}+\mathrm{SOD}+\mathrm{CAT}$ group (Figure 4(a)).

3.4. Increase of the Serum Levels of Renalase and Liver Enzymes in Hepatic I/R Injury Mouse Models and the Suppression to the Increased Renalase and Liver Enzymes by Antioxidants. The liver enzymes (ALT, AST, GGT, ALP, and $\mathrm{LDH})$ and renalase levels were measured in the serum of the hepatic I/R injury mouse models. The renalase levels were found to be significantly augmented in the hepatic I/R process $(p<0.01)$. The levels of ALT $(p<0.01)$, AST $(p<0.01)$, $\operatorname{ALP}(p<0.05)$, and LDH $(p<0.01)$ elevated significantly in the hepatic I/R group compared to the sham group, whereas GGT increased slightly in the hepatic I/R group and with no statistical significance to the sham group $(p>0.05)$. The increase of ALT, AST, LDH, and renalase levels could be effectively suppressed (renalase, ALT, and LDH: $p<0.01$; AST: $p<0.05)$ by the intraperitoneal preinjection of SOD and CAT. ALP and GGT could also be suppressed by antioxidants, but with no statistical significance $(p>0.05)$ to the I/R group (Figure 5).

\section{Discussion}

Hepatic I/R injury may occur in hepatic resections, liver transplantations, traumas, and vascular surgeries [33-35]. It is a serious complication in clinical practice. The redox balance in the process of hepatic I/R injury leads to accumulation of ROS, which presents the disease mechanism most commonly invoked in hepatic I/R injury [33]. Suppressing ROS-burst in oxidative stress is an efficient way to alleviate hepatic I/R injury. However, early warning, evaluation of the severity, or therapeutic effect of oxidative stress, which is even more important in clinical practice of hepatic I/R injury 


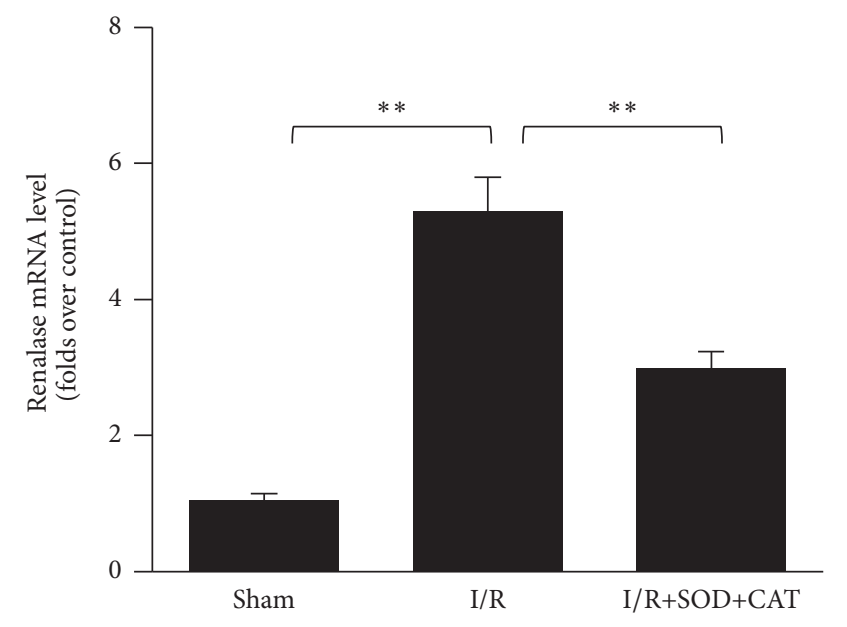

(a)

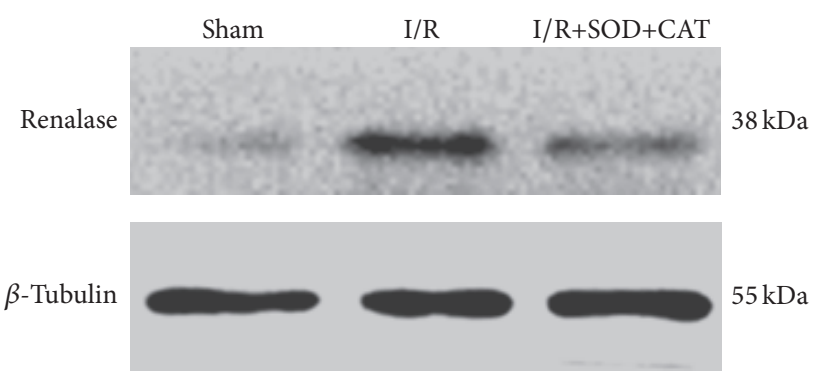

(b)

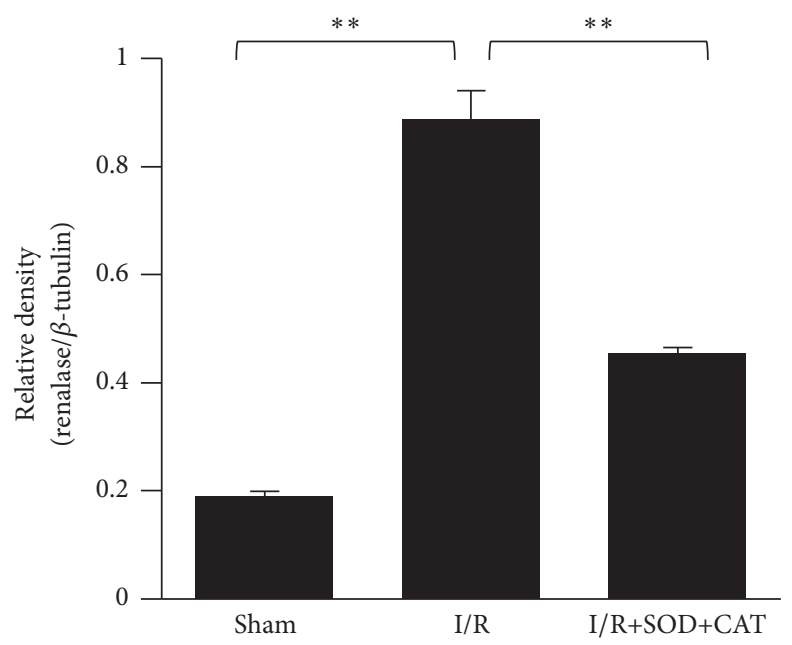

(c)

FIGURE 3: Augmentation of renalase expression in hepatic I/R injury models and the subsequent decrease induced by antioxidant preinjection. (a) Relative expression of renalase mRNA evaluated by real-time qPCR in livers of mouse models (sham, I/R injury, and I/R injury with antioxidant preinjection, $6 \mathrm{~h}$ after reperfusion). (b) Western blot analysis of renalase protein levels in livers of mouse models (sham, I/R injury, and I/R injury with antioxidant preinjection, $6 \mathrm{~h}$ after reperfusion). (c) Densitometric analysis of Western blot of renalase in livers of mouse models (sham, I/R injury, and I/R injury with antioxidant preinjection, $6 \mathrm{~h}$ after reperfusion). ${ }^{* *} p<0.01$. Data are plotted as the mean \pm SD from five independent experiments. Bars indicate the standard deviation of the mean.

management, is a remaining challenge. This is due to the lack of efficient, specific, and sensitive biomarkers for the accurate evaluation of the severity of oxidative stress [19]. Biochemical markers used to evaluate the severity of oxidative stress include malondialdehyde (MDA) [36, 37], ascorbic acid (AA)/dehydroascorbic acid (DHA) $[38,39]$, and a series of inflammatory, proinflammatory, or anti-inflammatory biomarkers [40-42]. However, these markers face various limitations. MDA is a marker for lipid peroxidation, which usually proceeds in the I/R injury of the extremities [43]. A close relationship between MDA and cardiac necrosis markers has been reported [36], but whether the MDA level can reflect the severity of oxidative stress or I/R injury of other organs remains unclear. The determination of AA and DHA levels is challenging because of the unstable nature of these compounds [39]. Inflammatory, proinflammatory, and anti-inflammatory markers cover a wide range of cytokines. However, these cytokines are not specific for evaluating oxidative stress. Therefore, an efficient, specific, and sensitive biomarker for evaluating oxidative stress is still urgently needed.

The human renalase gene is located on chromosome 10 at q23.33 and encodes the conserved protein renalase consisting of 342 amino acids. Renalase was reported to efficiently degrade dopamine and epinephrine in the blood [21], and it has been verified that it is the only enzyme involved in the catecholamine metabolism that can be secreted into the blood cycle hitherto [44]. Previous studies indicated that renalase is multifunctional and closely related to oxidative stress conditions such as stroke, heart transplantation, or acute 

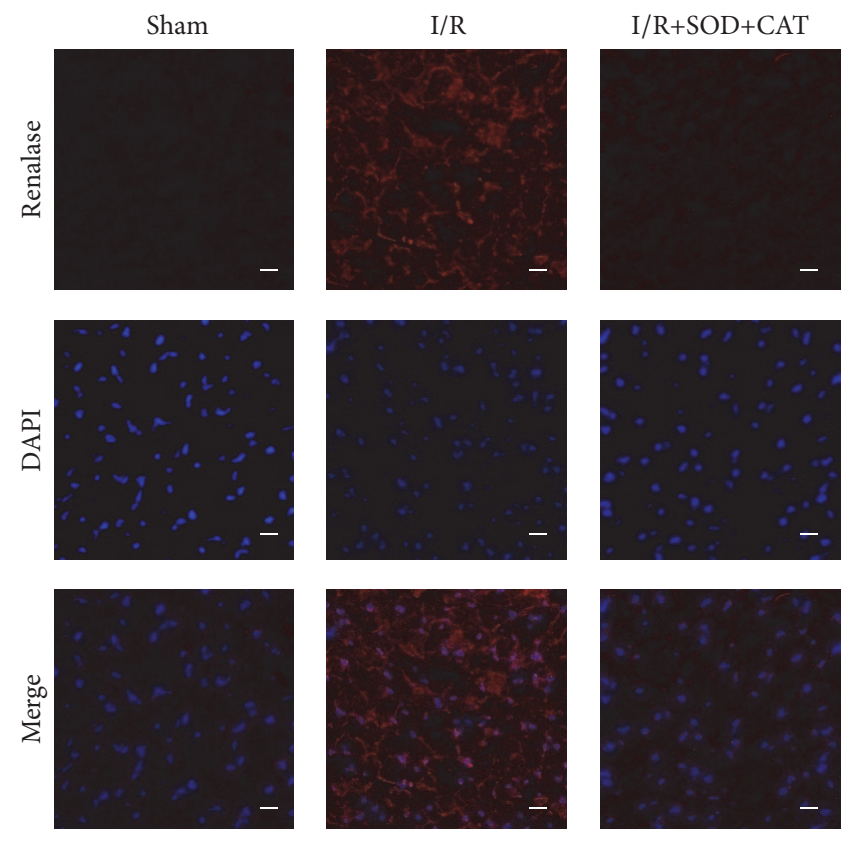
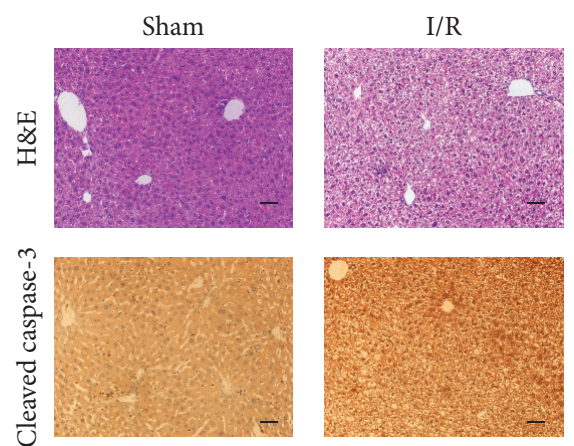

(a)

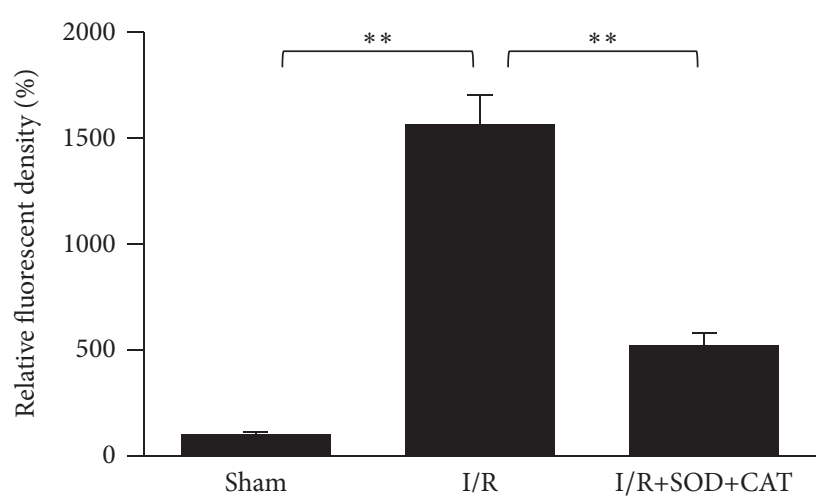

(b)

FIGURE 4: Increase of renalase levels in hepatic I/R injury models and its suppression induced by antioxidant preinjection and the corresponding histological changes in liver. (a) The livers of the sham, I/R, and IR+SOD+CAT groups ( $6 \mathrm{~h}$ after reperfusion) were subjected to histological evaluation by H\&E staining and IHC of cleaved caspase-3. Scale bar (black) represents $50 \mu \mathrm{m}$. (b) Confocal immunofluorescence imaging of renalase in livers of mouse models (sham, I/R injury, and I/R injury with antioxidant preinjection, $6 \mathrm{~h}$ after reperfusion). Scale bar (white) represents $30 \mu \mathrm{m}$. The relative fluorescent density of each group was analyzed. ${ }^{* *} p<0.01$. Data are plotted as the mean \pm SD from five independent experiments. Bars indicate the standard deviation of the mean.

kidney injury [45-48]. Considering its tight relationship with oxidative stress, renalase is thought to play a role, or at least responsive, in the process of hepatic I/R injury.

In particular, $\mathrm{H}_{2} \mathrm{O}_{2}$ is considered as the most abundant and stable ROS. $\mathrm{H}_{2} \mathrm{O}_{2}$ is a mild oxidant but is converted into hydroxyl radicals, which are extremely reactive and more toxic than other ROS $[49,50]$. Therefore, to investigate the responsiveness of renalase in hepatic cells under the condition of oxidative stress, $\mathrm{H}_{2} \mathrm{O}_{2}$ was used to mimic the ROSburst in oxidative stress. For the cell model in vitro, the common hepatocellular carcinoma cell line HepG2 was chosen. Renalase expression in HepG2 cells showed a positive correlation with $\mathrm{H}_{2} \mathrm{O}_{2}$ concentration and incubation time. These results suggest that renalase is responsive to the ROS-burst or oxidative stress in hepatic cells, and it is sensitive to the degree or severity and duration of oxidative stress.

Disturbance of the redox balance is characteristic for oxidative stress in the process of the hepatic I/R injury, which leads to ROS-burst and antioxidant consumption [33]. The most efficient way to ameliorate oxidative stress is replenishing ROS-scavenging antioxidants [17]. SOD is a common antioxidant, which is wildly distributed within mammalian organisms [51]. It is an important free radical scavenger, which has been clinically used in several countries. CAT is also an endogenous antioxidant, which can catalytically decompose $\mathrm{H}_{2} \mathrm{O}_{2}[52,53]$, but has not been used in clinical practice. In this study, SOD and CAT were used for the preincubation of the cells. We observed that 

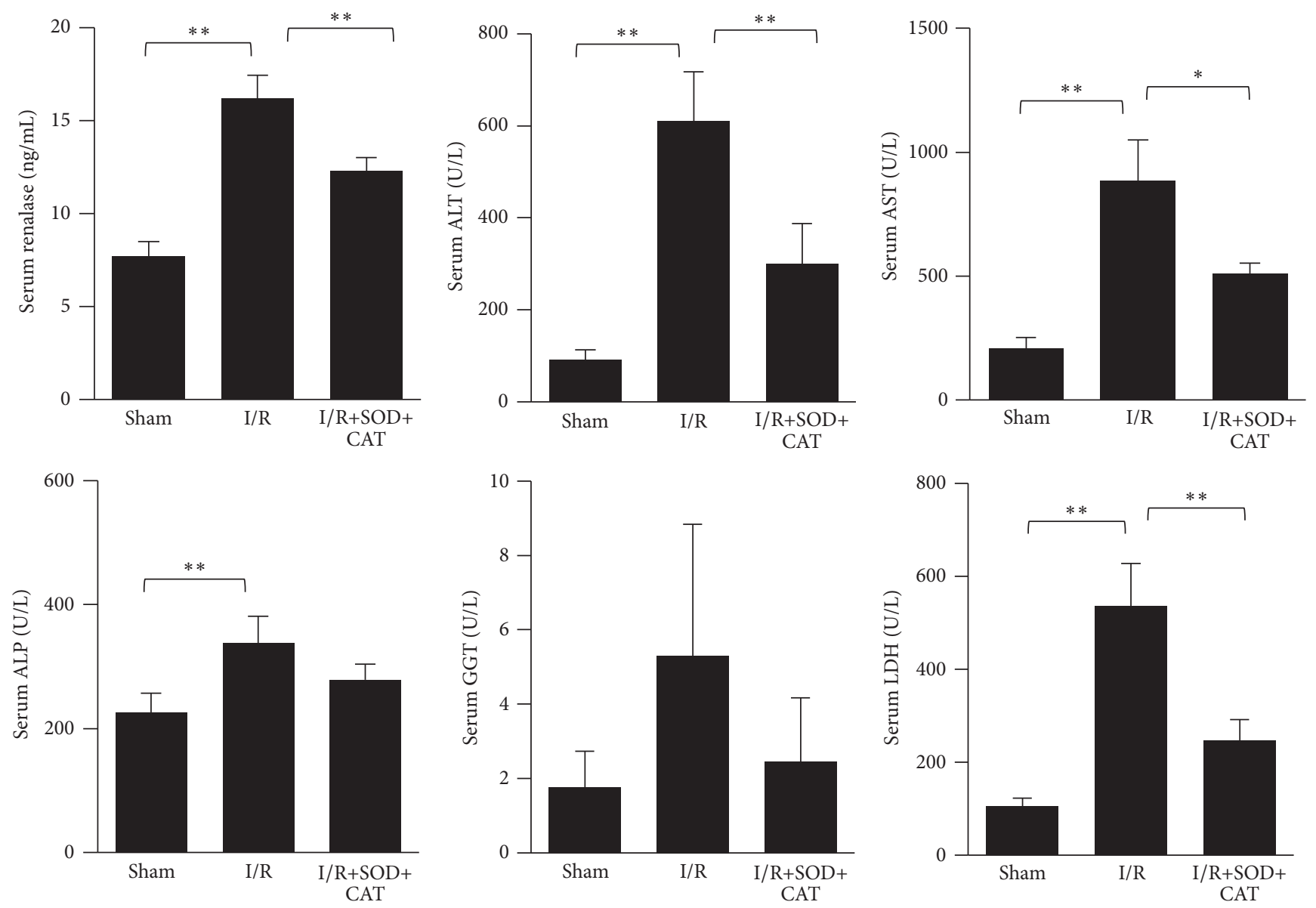

FiguRe 5: Increase of serum renalase and liver enzymes levels in hepatic I/R injury models and its suppression induced by antioxidant preinjection. Serum renalase and liver enzymes: ALT, AST, GGT, ALP, and LDH levels in mouse models (sham, I/R injury, and I/R injury with antioxidant preinjection, $6 \mathrm{~h}$ after reperfusion). ${ }^{*} p<0.05 ;{ }^{* *} p<0.01$. Data are plotted as the mean \pm SD from five independent experiments. Bars indicate the standard deviation of the mean.

the SOD/CAT preincubation significantly ameliorated the elevation of the renalase expression in HepG2 cells. The application of antioxidants reduced the severity of oxidative stress, and the responsive elevation of the renalase expression was ameliorated accordingly. This result suggests that renalase is responsive to the therapeutic means for oxidative stress in hepatic cells. And the expression of renalase is closely related to the degree or severity of the oxidative stress.

The hepatic I/R injury mouse model is the most commonly used animal model in various studies for hepatic I/R injury and oxidative stress in vivo. We used this model to investigate whether renalase is responsive to the hepatic $\mathrm{I} / \mathrm{R}$ injury in vivo. Renalase expression was significantly increased in the livers of hepatic I/R injury mouse models. Intraperitoneal preinjection of SOD and CAT ameliorated the elevation of renalase in the livers significantly. Furthermore, a consistent high-and-low variation between the renalase expression in liver and the degree of liver damage, including tissue necrosis and cell apoptosis, has been observed. Liver enzymes are the most important evaluation indexes in the standard liver function test (LFT) panel. Serum levels of liver enzymes, including ALT, AST, GGT, ALP, and LDH, were increased by different degrees in the hepatic I/R injury mouse models. Consistently, the serum renalase levels of the hepatic I/R injury mouse models were significantly elevated. The elevations of serum levels of either the renalase or the liver enzymes could be significantly reduced by the intraperitoneal preinjection of SOD and CAT. These results suggest that renalase is responsive to hepatic $\mathrm{I} / \mathrm{R}$ injury and therapeutic means. Its expression in liver tissue closely related to the severity of oxidative stress and the subsequent liver damage. Moreover, the serum renalase level can sensitively reflect the severity and therapeutic effect of oxidative stress in the process of hepatic I/R injury. Combining with the standard LFT panel, more accurate and specific evaluations of the hepatic I/R injury can be made.

In conclusion, to the best of our knowledge, this study firstly demonstrates the value and applicability of renalase as a promising biological marker for the evaluation of the severity of oxidative stress in hepatic I/R injury. We investigated the responsiveness of renalase to conditions related to oxidative stress in vitro and in vivo. Our results revealed that oxidative stress efficiently induces the elevation of renalase expression in vitro and in vivo. Furthermore, this elevated 
renalase expression can be decreased by antioxidants. Its sensitive responsiveness to the severity of oxidative stress and convenient detection in the blood make renalase an ideal biomarker for the evaluation of the severity and therapeutic effect of hepatic I/R injury. In the future, clinical treatment decisions based on the detection of renalase activities in the blood may help to improve the clinical outcomes of hepatic resections, liver transplantations, traumas, or vascular surgeries. Nevertheless, the variation trend of serum renalase level in the hepatic I/R process under different conditions and time points has not been demonstrated in this study, which needs further investigations. Beside the I/R injury, there are various pathophysiological processes which are related to oxidative stress. Renalase may play roles in these processes, and the variation trend of renalase expression in these processes may be different from each other. Related researches may make renalase serve as a novel inspection item, which can assist the existing inspection items to make the evaluations of the diseases more accurate. Other potential applications of renalase in clinical practice require further explorations and investigations.

\section{Disclosure}

The funders had no role in study design, data collection and analysis, decision to publish, or preparation of the manuscript.

\section{Competing Interests}

The authors declare that there are no competing interests regarding the publication of this paper.

\section{Acknowledgments}

This work was financially supported by the Scientific Research Training Program for Young Talents from Union Hospital of Tongji Medical College, HUST (to Huili Li); by the Natural Science Foundation of China (81602419 to Huili Li); by the Natural Science Foundation of China ( 81500348 to Kun Huang); by the Independent Innovation Foundation of HUST (0118530218 to Huili Li); by the Natural Science Foundation of China (81570568 to Jiliang Wang); by the Research Fund of Public Welfare in Health Industry, 2014, Health Ministry of China (201402015 to Guobin Wang); by the Natural Science Foundation of Hubei Province (2013CFB131 to Lixia Wang); by the Foundation of Health and Family Planning Commission of Hubei Province (WJ2015MB075 to Yanfeng Niu).

\section{References}

[1] M. Glanemann, R. Strenziok, R. Kuntze et al., "Ischemic preconditioning and methylprednisolone both equally reduce hepatic ischemia/reperfusion injury," Surgery, vol. 135, no. 2, pp. 203-214, 2004.

[2] D. L. Carden and D. N. Granger, "Pathophysiology of ischaemia-reperfusion injury," The Journal of Pathology, vol. 190, no. 3, pp. 255-266, 2000.
[3] C. S. Kunak, O. Kukula, E. Mutlu et al., "The effect of etoricoxib on hepatic ischemia-reperfusion injury in rats," Oxidative Medicine and Cellular Longevity, vol. 2015, Article ID 598162, 8 pages, 2015.

[4] J. Yu, H.-S. Lee, S.-M. Lee et al., "Aggravation of post-ischemic liver injury by overexpression of A20, an NF- $\kappa$ B suppressor," Journal of Hepatology, vol. 55, no. 2, pp. 328-336, 2011.

[5] W. Zhang, M. Wang, H. Y. Xie et al., "Role of reactive oxygen species in mediating hepatic ischemia-reperfusion injury and its therapeutic applications in liver transplantation," Transplantation Proceedings, vol. 39, no. 5, pp. 1332-1337, 2007.

[6] C. Kang, W. Cho, M. Park et al., " $\mathrm{H}_{2} \mathrm{O}_{2}$-triggered bubble generating antioxidant polymeric nanoparticles as ischemia/ reperfusion targeted nanotheranostics," Biomaterials, vol. 85, pp. 195-203, 2016.

[7] J. Lutz, K. Thürmel, and U. Heemann, "Anti-inflammatory treatment strategies for ischemia/reperfusion injury in transplantation," Journal of Inflammation, vol. 7, article 27, 2010.

[8] G. C. Van de Bittner, E. A. Dubikovskaya, C. R. Bertozzi, and C. J. Chang, "In vivo imaging of hydrogen peroxide production in a murine tumor model with a chemoselective bioluminescent reporter," Proceedings of the National Academy of Sciences of the United States of America, vol. 107, no. 50, pp. 21316-21321, 2010.

[9] E. W. Miller, A. E. Albers, A. Pralle, E. Y. Isacoff, and C. J. Chang, "Boronate-based fluorescent probes for imaging cellular hydrogen peroxide," Journal of the American Chemical Society, vol. 127, no. 47, pp. 16652-16659, 2005.

[10] D. Lee, S. Bae, Q. Ke et al., "Hydrogen peroxide-responsive copolyoxalate nanoparticles for detection and therapy of ischemia-reperfusion injury," Journal of Controlled Release, vol. 172, no. 3, pp. 1102-1110, 2013.

[11] Y. Deng, L. Zhao, and X. Lu, "A multidrug cocktail approach attenuates ischemic-type biliary lesions in liver transplantation from non-heart-beating donors," Medical Hypotheses, vol. 91, pp. 47-52, 2016.

[12] F. Chamoun, M. Burne, M. O’Donnell, and H. Rabb, "Pathophysiologic role of selectins and their ligands in ischemia reperfusion injury," Frontiers in Bioscience, vol. 5, pp. E103-E109, 2000.

[13] M. D. Wheeler, "Endotoxin and kupffer cell activation in alcoholic liver disease," Alcohol Research \& Health, vol. 27, no. 4, pp. 300-306, 2003.

[14] S.-F. Ma, M. Nishikawa, H. Katsumi, F. Yamashita, and M. Hashida, "Liver targeting of catalase by cationization for prevention of acute liver failure in mice," Journal of Controlled Release, vol. 110, no. 2, pp. 273-282, 2006.

[15] H. Katsumi, M. Nishikawa, H. Yasui, F. Yamashita, and M. Hashida, "Prevention of ischemia/reperfusion injury by hepatic targeting of nitric oxide in mice," Journal of Controlled Release, vol. 140, no. 1, pp. 12-17, 2009.

[16] D. Lee, S. Bae, D. Hong et al., " $\mathrm{H}_{2} \mathrm{O}_{2}$-responsive molecularly engineered polymer nanoparticles as ischemia/reperfusiontargeted nanotherapeutic agents," Scientific Reports, vol. 3, article 2233, 2013.

[17] D. Lee, S. Park, S. Bae et al., "Hydrogen peroxide-activatable antioxidant prodrug as a targeted therapeutic agent for ischemia-reperfusion injury," Scientific Reports, vol. 5, article 16592, 2015. 
[18] B. Suleyman, A. Albayrak, N. Kurt, E. Demirci, C. Gundogdu, and M. Aksoy, "The effect of etoricoxib on kidney ischemiareperfusion injury in rats: a biochemical and immunohistochemical assessment," International Immunopharmacology, vol. 23, no. 1, pp. 179-185, 2014.

[19] N. L. Halladin, "Oxidative and inflammatory biomarkers of ischemia and reperfusion injuries," Danish Medical Journal, vol. 62, no. 4, p. B5054, 2015.

[20] M. Du, K. Huang, D. Huang et al., "Renalase is a novel target gene of hypoxia-inducible factor-1 in protection against cardiac ischaemia-reperfusion injury," Cardiovascular Research, vol. 105, no. 2, pp. 182-191, 2015.

[21] J. Xu, G. Li, P. Wang et al., "Renalase is a novel, soluble monoamine oxidase that regulates cardiac function and blood pressure," The Journal of Clinical Investigation, vol. 115, no. 5, pp. 1275-1280, 2005.

[22] M. Buraczynska, P. Zukowski, K. Buraczynska, S. Mozul, and A. Ksiazek, "Renalase gene polymorphisms in patients with type 2 diabetes, hypertension and stroke," NeuroMolecular Medicine, vol. 13, no. 4, pp. 321-327, 2011.

[23] H. T. Lee, J. Y. Kim, M. Kim et al., "Renalase protects against ischemic AKI," Journal of the American Society of Nephrology, vol. 24, no. 3, pp. 445-455, 2013.

[24] P. Przybylowski, E. Koc-Zorawska, J. S. Malyszko, M. Mysliwiec, and J. Malyszko, "Renalase and endothelial dysfunction in heart transplant recipients," Transplantation Proceedings, vol. 45, no. 1, pp. 394-396, 2013.

[25] Y. Abe, I. N. Hines, G. Zibari et al., "Mouse model of liver ischemia and reperfusion injury: method for studying reactive oxygen and nitrogen metabolites in vivo," Free Radical Biology \& Medicine, vol. 46, no. 1, pp. 1-7, 2009.

[26] H. Li, K. Huang, X. Liu et al., "Lithium chloride suppresses colorectal cancer cell survival and proliferation through ROS/GSK-3 $\beta / \mathrm{NF}-\kappa \mathrm{B}$ signaling pathway," Oxidative Medicine and Cellular Longevity, vol. 2014, Article ID 241864, 8 pages, 2014.

[27] J.-H. Shin, K. S. Chun, Y.-G. Na et al., "Allopurinol protects against ischemia/reperfusion-induced injury in rat urinary bladders," Oxidative Medicine and Cellular Longevity, vol. 2015, Article ID 906787, 8 pages, 2015.

[28] Q. Yang, W. Wu, Q. Li et al., "High-dose polymerized hemoglobin fails to alleviate cardiac ischemia/reperfusion injury due to induction of oxidative damage in coronary artery," Oxidative Medicine and Cellular Longevity, vol. 2015, Article ID 125106, 10 pages, 2015.

[29] J. Bai, Y. Zheng, G. Wang, and P. Liu, "Protective effect of D-limonene against oxidative stress-induced cell damage in human lens epithelial cells via the p38 pathway," Oxidative Medicine and Cellular Longevity, vol. 2016, Article ID 5962832, 12 pages, 2016.

[30] K. Yanar, U. Çakatay, S. Aydin et al., "Relation between endothelial nitric oxide synthase genotypes and oxidative stress markers in larynx cancer," Oxidative Medicine and Cellular Longevity, vol. 2016, Article ID 4985063, 8 pages, 2016.

[31] L. A. Moreno-Ruíz, D. Ibarra-Quevedo, E. Rodríguez-Martínez et al., "Oxidative stress state is associated with left ventricular mechanics changes, measured by speckle tracking in essential hypertensive patients," Oxidative Medicine and Cellular Longevity, vol. 2015, Article ID 502107, 8 pages, 2015.

[32] H. Xie, J. Sun, Y. Chen, M. Zong, S. Li, and Y. Wang, "EGCG attenuates uric acid-induced inflammatory and oxidative stress responses by medicating the NOTCH pathway," Oxidative Medicine and Cellular Longevity, vol. 2015, Article ID 214836, 10 pages, 2015.

[33] M. Elias-Miro, M. B. Jimenez-Castro, J. Rodes, and C. Peralta, "Current knowledge on oxidative stress in hepatic ischemia/reperfusion," Free Radical Research, vol. 47, no. 8, pp. 555-568, 2013.

[34] N. C. Teoh, "Hepatic ischemia reperfusion injury: contemporary perspectives on pathogenic mechanisms and basis for hepatoprotection-the good, bad and deadly," Journal of Gastroenterology and Hepatology, vol. 26, no. 1, pp. 180-187, 2011.

[35] H. K. Eltzschig and C. D. Collard, "Vascular ischaemia and reperfusion injury," British Medical Bulletin, vol. 70, pp. 71-86, 2004.

[36] A. Pinelli, S. Trivulzio, S. Brenna, and G. Rossoni, "Plasma cardiac necrosis markers C-troponin i and creatine kinase, associated with increased malondialdehyde levels, induced in rabbits by means of 5-aminolevulinic acid injection," Pharmacology, vol. 84, no. 5, pp. 314-321, 2009.

[37] J. Lykkesfeldt, "Determination of malondialdehyde as dithiobarbituric acid adduct in biological samples by HPLC with fluorescence detection: comparison with ultraviolet-visible spectrophotometry," Clinical Chemistry, vol. 47, no. 9, pp. 1725-1727, 2001.

[38] H.-A. Lehr, B. Frei, A. M. Olofsson, T. E. Carew, and K.E. Arfors, "Protection from oxidized LDL-induced leukocyte adhesion to microvascular and macrovascular endothelium in vivo by vitamin C but not by vitamin E," Circulation, vol. 91, no. 5, pp. 1525-1532, 1995.

[39] J. Lykkesfeldt, S. Loft, and H. E. Poulsen, "Determination of ascorbic acid and dehydroascorbic acid in plasma by high- performance liquid chromatography with coulometric detectionare they reliable biomarkers of oxidative stress?" Analytical Biochemistry, vol. 229, no. 2, pp. 329-335, 1995.

[40] C. A. Dinarello, "Proinflammatory cytokines," Chest, vol. 118, no. 2, pp. 503-508, 2000.

[41] S. M. Opal and V. A. DePalo, "Anti-inflammatory cytokines," Chest, vol. 117, no. 4, pp. 1162-1172, 2000.

[42] I. Guillen, M. Blanes, M.-J. Gomez-Lechon, and J. V. Castell, "Cytokine signaling during myocardial infarction: sequential appearance of IL-1 beta and IL-6," The American Journal of Physiology - Regulatory Integrative and Comparative Physiology, vol. 269, no. 2, part 2, pp. R229-R235, 1995.

[43] A. Valenzuela, "The biological significance of malondialdehyde determination in the assessment of tissue oxidative stress," Life Sciences, vol. 48, no. 4, pp. 301-309, 1991.

[44] G. R. Moran, "The catalytic function of renalase: a decade of phantoms," Biochimica et Biophysica Acta-Proteins and Proteomics, vol. 1864, no. 1, pp. 177-186, 2016.

[45] M. T. Wybraniec and K. Mizia-Stec, "Renalase and biomarkers of contrast-induced acute kidney injury," CardioRenal Medicine, vol. 6, no. 1, pp. 25-36, 2015.

[46] J. Malyszko, H. Bachorzewska-Gajewska, and S. Dobrzycki, "Renalase, kidney and cardiovascular disease: are they related or just coincidentally associated?" Advances in Medical Sciences, vol. 60, no. 1, pp. 41-49, 2015.

[47] G. V. Desir and A. J. Peixoto, "Renalase in hypertension and kidney disease," Nephrology Dialysis Transplantation, vol. 29, no. 1, pp. 22-28, 2014.

[48] X. Guo, L. Wang, H. Velazquez, R. Safirstein, and G. V. Desir, "Renalase: its role as a cytokine, and an update on its association 
with type 1 diabetes and ischemic stroke," Current Opinion in Nephrology and Hypertension, vol. 23, no. 5, pp. 513-518, 2014.

[49] M. Ushitora, F. Sakurai, T. Yamaguchi et al., "Prevention of hepatic ischemia-reperfusion injury by pre-administration of catalase-expressing adenovirus vectors," Journal of Controlled Release, vol. 142, no. 3, pp. 431-437, 2010.

[50] T. Hanawa, S. Asayama, T. Watanabe, S. Owada, and H. Kawakami, "Protective effects of the complex between manganese porphyrins and catalase-poly(ethylene glycol) conjugates against hepatic ischemia/reperfusion injury in vivo," Journal of Controlled Release, vol. 135, no. 1, pp. 60-64, 2009.

[51] J. M. McCord and I. Fridovich, "Superoxide dismutase. An enzymic function for erythrocuprein (hemocuprein)," The Journal of Biological Chemistry, vol. 244, no. 22, pp. 6049-6055, 1969.

[52] D. W. May, "Catalase, a new enzym of general occurrence," Science, vol. 14, no. 360, pp. 815-816, 1901.

[53] C. A. Evans, "On the catalytic decomposition of hydrogen peroxide by the catalase of blood," The Biochemical Journal, vol. 2, no. 4, pp. 133-155, 1907. 


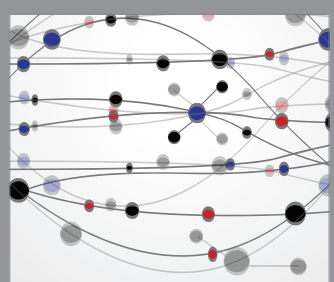

The Scientific World Journal
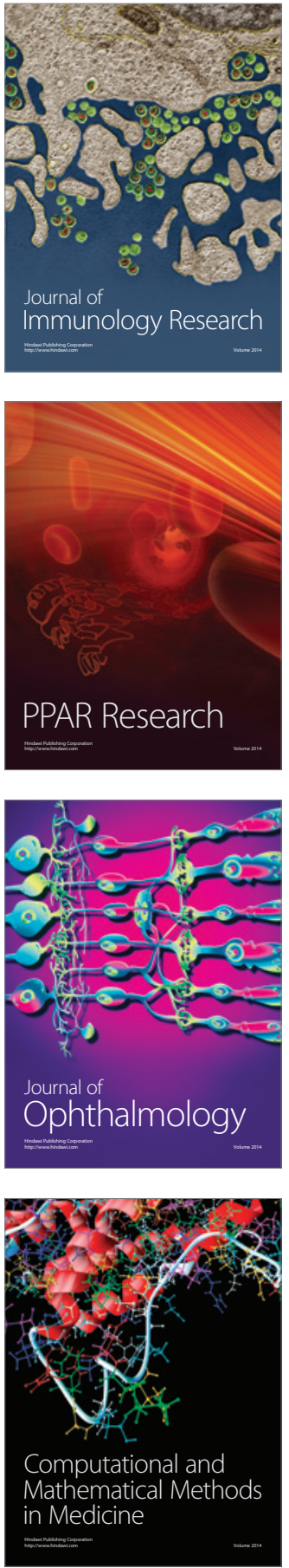

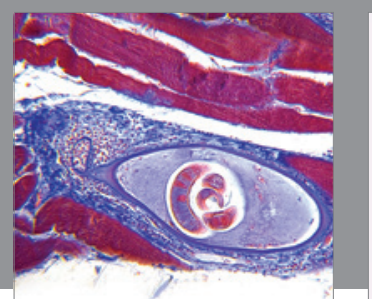

Gastroenterology Research and Practice

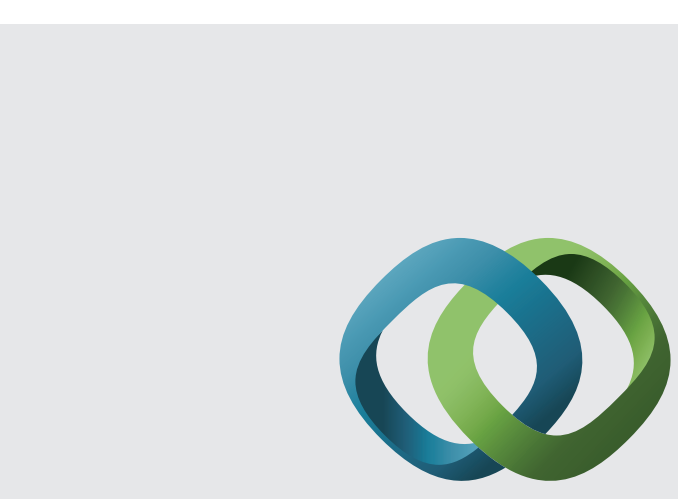

\section{Hindawi}

Submit your manuscripts at

http://www.hindawi.com
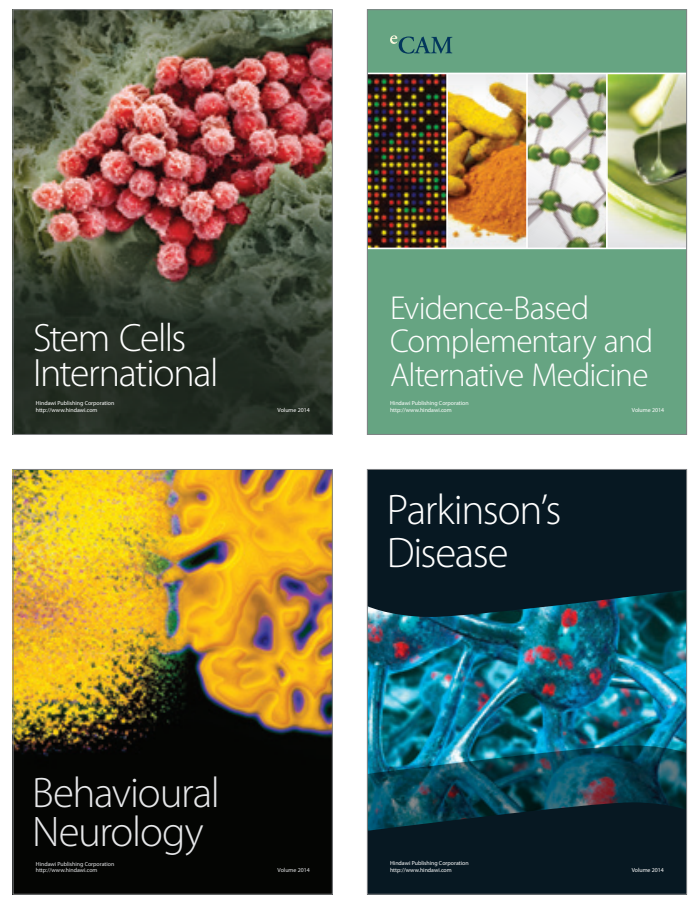
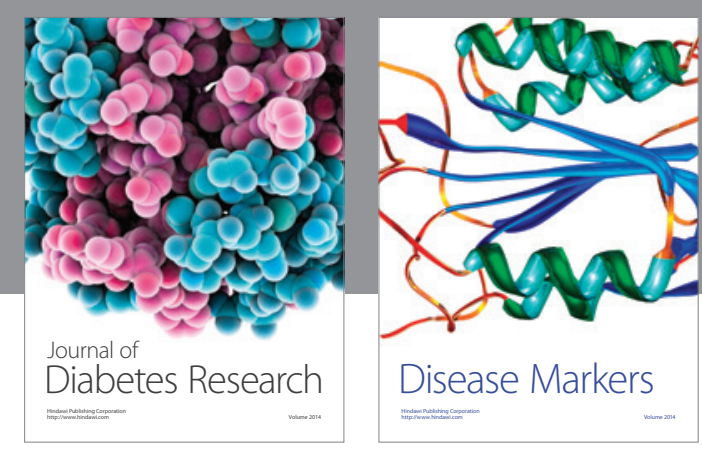

Disease Markers
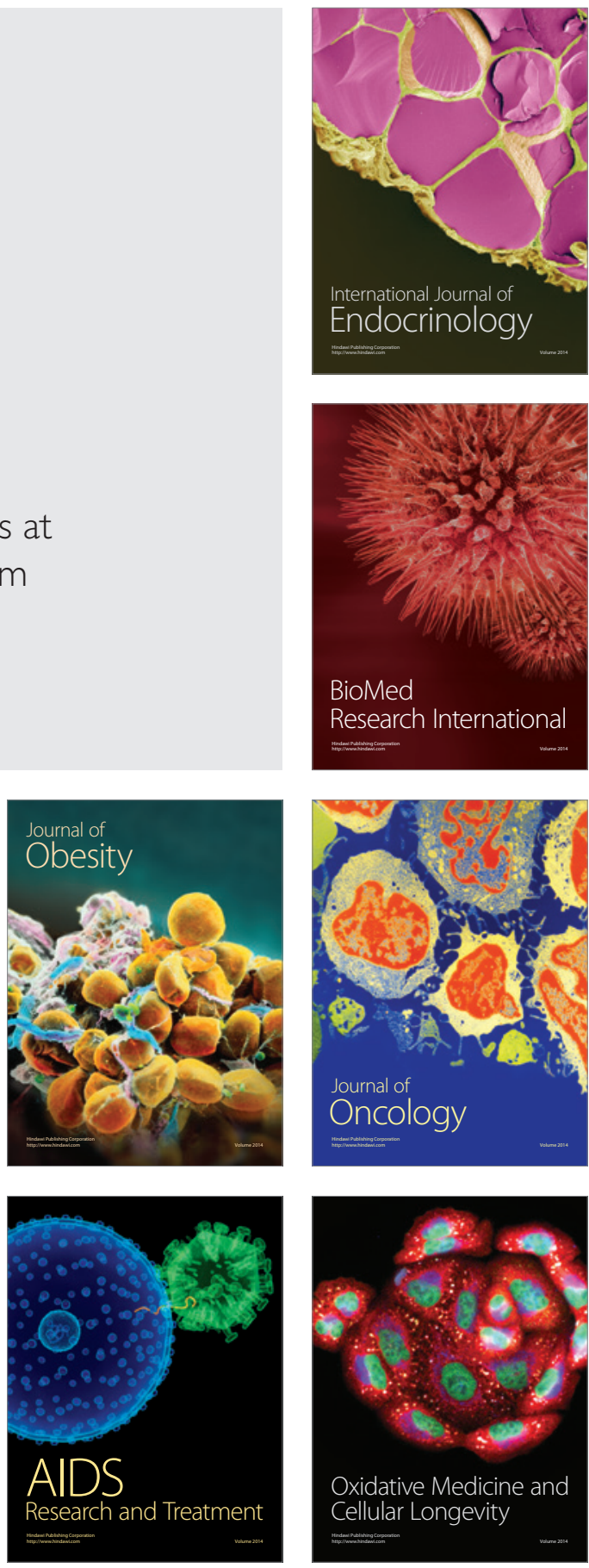